\title{
Índice de Sharpe e outros Indicadores de Performance Aplicados a Fundos de Ações Brasileiros
}

\author{
Gyorgy Varga
}

\section{RESUMO}

Implementamos e aplicamos diversas medidas estatísticas de avaliação de performance aos dez maiores fundos de ações oferecidos no mercado brasileiro. Mostramos as diferentes classificações dos fundos de acordo com cada um desses indicadores e os defeitos da sua aplicação na avaliação de fundos de investimento. O principal objetivo deste artigo é mostrar as dificuldades da aplicação dos principais indicadores de performance a fundos brasileiros.

Palavras-chaves: investimentos; finanças; modelos de performance.

\begin{abstract}
We applied several performance measures to evaluate the ten largest stock mutual funds offered in Brazil. The main purpose of this article is to introduce and show the difficulties and shortfalls on the application of the performance measures to the brazilian mutual fund industry.
\end{abstract}

Key words: investments; finance; portfolio performance. 


\section{INTRODUÇÃO}

O processo de seleção de um fundo de investimento pode ser dividido em duas partes. A primeira verifica qual a carteira adequada para o investidor; a segunda tenta descobrir um gestor que pode superar o mercado. Se não for encontrado nenhum, então o caminho natural é um fundo passivo que apenas acompanhe as classes de ativos escolhidas. O investimento em fundo passivo é a referência e a alternativa mais adequada, quando não há um bom gestor ativo conhecido ${ }^{(1)}$.

O gestor capaz de superar o mercado é aquele que tem acesso a melhores informações, e/ou tem modelos para mais bem processar as informações disponíveis para o público em geral. É o conjunto de informação e/ou seus modelos que permitem ao bom gestor proporcionar um retorno superior ao do mercado, justificando assim a administração ativa. Em troca do retorno superior esperado, o gestor ativo costuma cobrar taxas de administração e de performance maiores do que os fundos de gestão passiva; portanto só vale a pena incorrer nesse custo maior, quando há forte evidência de que o gestor ativo tem condição de superar o mercado.

Os praticantes desse mercado e as publicações especializadas tentam classificar e apontar os melhores fundos de investimento e também avaliar a contribuição do gestor com base em procedimentos estatísticos retirados dos modernos modelos de finanças. Esses procedimentos são inspirados especialmente no modelo $\mathrm{CAPM}^{(2)}$, que postula certa relação entre risco e retorno. Outros modelos de risco para o mercado financeiro, tal como o $\mathrm{APT}^{(3)}$, também podem ser usados para se avaliar a performance de investimento, como pode ser visto em qualquer manual de finanças atual, como, por exemplo, Haugen (1997). Nas seções seguintes, mostramos algumas estatísticas simples de avaliação de performance, como a média do retorno; estatísticas inspiradas no CAPM (entre elas o famoso índice de Sharpe), e outros procedimentos que permitem melhor avaliação do timing de uma carteira ativa e também do sucesso da gestão passiva.

A principal adição dos modelos de finanças à avaliação dos fundos vem da incorporação do risco, que se iniciou com o modelo de média-variância de Markowitz, na década de 50. Se o retorno esperado de um fundo é tanto maior quanto maior seu risco, então a inclusão de alguma medida de risco na avaliação deste permite melhor verificação de quanto do retorno proporcionado por um gestor veio do risco assumido e quanto veio de seu talento ou sorte. Assim, fica mais bem explicada a verdadeira contribuição do gestor para o retorno do fundo. 
Outro aspecto importante na inclusão do risco é a sua contribuição para a determinação da carteira ótima de um investidor (para investidores que se importem com risco, porquanto, no caso de investidores neutros ao risco, basta conhecer o retorno esperado).

$\mathrm{Na}$ avaliação de fundos de investimento, muitos praticantes e publicações especializadas tomam as estatísticas dos modelos acima, produzidas com base em dados históricos, como se fossem bons estimadores do futuro desempenho do fundo. Eles assumem a hipótese heróica de que séries de preços passados permitem boas estimativas para os preços futuros, daí o chavão de alguns: "retornos passados não são garantia de retornos futuros. "Ainda que tivéssemos boas estimativas para o risco e retorno, um resultado interessante, apresentado por Admati e Ross (1985), destaca que medidas de performance que dão melhor qualidade a gestores com maior relação retorno/risco não indicam corretamente quais são os gestores mais bem informados, pois esses certamente irão alterar bastante suas carteiras em função da maior quantidade de informação, gerando assim maior volatilidade (risco). No limite, um fundo que tem uma carteira estática certamente não tem um gestor com informação ou modelos superiores.

Entre as estatísticas de avaliação de performance mais conhecidas estão o índice de Sharpe (IS), apresentado em Sharpe (1966), que, desde então, tem sido amplamente utilizado na indústria de fundos de investimento. Apesar da sua importância prática, sua utilização depende da estimação correta dos seus parâmetros e da aplicação que se pretende. Na próxima seção, mostramos como calcular o retorno; a seguir discutimos a inclusão e avaliação do risco e, mostramos como calcular o IS e as falhas a que ele está sujeito; por fim mostramos outras medidas para comparar fundos e fazemos uma breve aplicação aos dez maiores fundos de ações negociados no Brasil.

Vale lembrar a importância desses indicadores, tanto para praticantes quanto para todo o tipo de investidor, pois esse é um instrumental disponível para a tomada de decisão dos investidores, e recursos vultosos mudam de mãos de acordo com o resultado desses números. Neste artigo mostram-se as falhas desses indicadores e como deve ser feita a sua aplicação. Toma-se como exemplo uma amostra de três anos, com dados semanais dos dez maiores fundos de ações brasileiros. Esperamos contribuir para as finanças nacionais, cobrindo uma lacuna na literatura e, possivelmente, ajudar na melhor alocação de recursos em nosso país. 


\section{RETORNO}

O indicador de performance mais simples e talvez o mais utilizado na prática é a taxa de retorno realizada. Mas a preocupação dos investidores deve ser com a taxa esperada (ex-ante) de retorno e não com o que já aconteceu (ex-post). Esse talvez seja o indicador mais importante para a maioria dos investidores (certamente o é para os investidores neutros a risco). Há enorme dificuldade em se obter esses valores esperados. Dessa forma, uma estimativa muito comum para o retorno esperado vem da avaliação da média do seu retorno histórico, de modo que, implicitamente, é suposto que o passado vai-se repetir de alguma forma.

No caso de fundos de investimento que não costumam ter nenhum pagamento intermediário, a taxa de retorno costuma ser definida como o valor final sobre o valor inicial da cota do fundo:

$$
r_{t} \frac{Q_{t}}{Q_{t 1}} \quad 1
$$

onde $Q_{t}$ é o valor da cota na data $t$.

Não obstante sua simplicidade, a estimativa do retorno médio obtido a partir de uma série histórica pode ser avaliada por diferentes procedimentos. Tomando-se uma série de cotações de um fundo, pode-se calcular a série de taxas de retornos periódicos, e daí se obtém sua média aritmética:

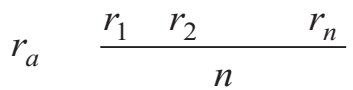

Alternativamente, pode-se tomar a média geométrica:

$$
\begin{array}{llllllll}
r_{g} & 1 & r_{1} & 1 & r_{2} & 1 & r_{n} & (1 / n) \\
1
\end{array}
$$

A média geométrica é financeiramente consistente, quando se trata de reconstituir o retorno de todo o período; mas, se procuramos o retorno esperado de um período intermediário, a média aritmética é a estatística correta ${ }^{(4)}$. É fácil mostrar que a média aritmética é tanto maior do que a geométrica, quanto maior for a volatilidade da taxa de retorno periódica.

A relação entre a média aritmética e geométrica é dada por: 


$$
r_{g} \quad r_{a} \quad 1 / 22^{2}
$$

Exemplo 1. Seja um fundo com as seguintes cotações anuais:

\begin{tabular}{c|c|c}
\hline Ano & Cota & Retorno \\
\hline 1 & 100 & \\
2 & 200 & $100 \%$ \\
3 & 200 & $0 \%$ \\
4 & 200 & $0 \%$ \\
5 & 100 & $-50 \%$ \\
\hline
\end{tabular}

A média geométrica do retorno é de $0 \%$ aa, e a aritmética é de $12,5 \%$ aa. A estimativa correta para a taxa de retorno esperada anual é de $12,5 \%$, isto é, baseada na média aritmética. Por exemplo, o retorno esperado do investimento de $\mathrm{R} \$ 100,00$, com base nos dados do Exemplo 1, por um ano apenas, é de $(100,00+0+0-50,00) / 4=12,50$, que equivale a uma taxa de retorno anual de $12,5 \%$, como indica a estimativa pela média aritmética.

A volatilidade anual da taxa de retorno é de 54\%. Da Equação [4] se obtém uma aproximação para a taxa de retorno geométrica de:

$$
r_{g} \quad 12,5 \% \quad 54 \%^{2} / 2
$$

Para ilustrar a diferença entre os dois procedimentos de cálculo sobre ativos conhecidos no Brasil, mostramos, no Quadro 1, o retorno do CDI e do índice Ibovespa entre janeiro e outubro de 2000. Devido à alta volatilidade, o retorno do Ibovespa estimado pela sua média aritmética é significativamente maior do que aquele estimado pela média geométrica.

\section{Quadro 1: Comparação do Retorno do CDI e Ibovespa}

\begin{tabular}{c|c|c}
\hline Retomo Mens al & CDI & Ibovesp \\
\hline Média aritmética & $1,39 \%$ & $1,54 \%$ \\
Média geométrica & $1,39 \%$ & $1,30 \%$ \\
Volatilidade (anual) & $0,3 \%$ & $25,7 \%$ \\
Retorno Total & $11,65 \%$ & $10,89 \%$ \\
\hline
\end{tabular}


A qualidade estatística das estimativas baseadas na série histórica é proporcional ao tamanho da amostra utilizada. Quanto maior o tamanho da amostra maior a confiança estatística na estimativa.

Exemplo 2. Tomamos a amostra de retornos diários de todo o ano de 1998 do fundo de ações do Banco do Brasil, totalizando 248 observações, e obtivemos seu retorno médio diário igual a $-0,19 \%$. Com $95 \%$ de probabilidade, tem-se um intervalo para a média de $[-0,47 \%$ e $0,09 \%]$. No mesmo período, a taxa média diária do CDI foi de $0,10 \%$; portanto, com alto grau de confiança estatística, o retorno estimado com base numa séria histórica forneceu uma estimativa sem muito sentido econômico, já que tem pouco sentido investir-se em um fundo de ações com retorno esperado negativo.

Outras maneiras de se obterem estimativas para o retorno esperado são: tomar o retorno esperado pelos analistas de mercado ou elaborar algum modelo econométrico baseado no APT ou CAPM.

\section{UM MOdelo de Risco}

Para os investidores que se importam com o risco do retorno de seus investimentos, alguma medida de risco deve ser incorporada na análise. O primeiro passo é determinar uma distribuição de probabilidade adequada para a taxa de retorno. A mais usual é a distribuição normal que arbitrariamente tomamos como apropriada para descrever o comportamento da taxa de retorno; entretanto boa parte dos investimentos financeiros não podem ser rigorosamente representados por uma normal, porque essa distribuição permite que a taxa de retorno seja menor do que $-100 \%$, e sabemos que boa parte dos investimentos têm passivo limitado. Uma alternativa seria atribuir a distribuição normal à taxa de retorno na sua forma contínua, que corresponde a uma distribuição lognormal para o valor do investimento; só que outras dificuldades técnicas surgiriam na determinação da carteira ótima. Além disso, para pequenos intervalos de tempo, a distribuição normal para a taxa de retorno simples é uma boa aproximação. Assim, as inferências sobre a taxa de retorno esperado serão feitas, supondo que ela tem distribuição normal.

O passo seguinte é estimar o desvio-padrão da taxa de retorno, igualmente conhecido como volatilidade. O objetivo também é obter valores ex-ante; novamente, costuma-se tomar a série histórica dos retornos para se gerar uma estimativa para a volatilidade esperada. Quanto à estimativa propriamente dita, di- 
versos métodos podem ser adotados, entre os quais o mais conhecido é o estimador de máxima verossimilhança, dado por:

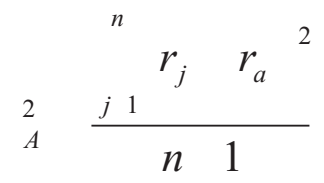

Essa estimativa pode ser facilmente feita em uma planilha Excel. Existem até mesmo funções que executam essa conta imediatamente. Outros métodos para o cálculo da volatilidade estão disponíveis, por exemplo: baseados nos valores máximos e mínimos das taxas de retorno (método de valores extremos), suavização exponencial (do riskmetrics) e métodos de séries de tempo do tipo Garch. Uma descrição desses procedimentos pode ser encontrada em Duarte e Pinheiro (1996).

Exemplo 3. Analisamos o retorno de uma amostra de fundos, com base em cotações semanais, dos anos de 1997, 1998 e 1999(5), de dez dos maiores fundos de ações oferecidos no mercado brasileiro. Esses dez fundos foram selecionados de acordo com o tamanho médio do seu patrimônio durante os três anos. Foram excluídos os fundos exclusivos e os fechados para captação. Para efeito de comparação, no final do Quadro 2 está a volatilidade dos índices Ibovespa, IBX e CDI.

\section{Quadro 2: Volatilidade Anual dos Dez Maiores Fundos de Ações}

\begin{tabular}{l|c|c|c} 
& \multicolumn{3}{c}{ Volatilidade anual } \\
& 1997 & 1998 & 1999 \\
\hline BB FUNDO DE AÇÕES & $39 \%$ & $48 \%$ & $33 \%$ \\
BOSTON AÇÕES & $44 \%$ & $62 \%$ & $37 \%$ \\
CCF-AÇÕES & $48 \%$ & $62 \%$ & $37 \%$ \\
CITIAÇÕES & $45 \%$ & $59 \%$ & $36 \%$ \\
ITAUAÇÕES & $35 \%$ & $39 \%$ & $34 \%$ \\
UNIBANCO BLUE & $45 \%$ & $52 \%$ & $35 \%$ \\
IP PARTICIPAÇÕES & $17 \%$ & $37 \%$ & $24 \%$ \\
BRADESCO CART. LIVRE & $46 \%$ & $62 \%$ & $37 \%$ \\
UNIBANCO STRATEGY & $43 \%$ & $54 \%$ & $36 \%$ \\
OPPORTUNITY LÓGICA II - CL & $46 \%$ & $47 \%$ & $53 \%$ \\
\hline CDI & $1 \%$ & $1 \%$ & $1 \%$ \\
Ibovesp & $43 \%$ & $54 \%$ & $33 \%$ \\
IBX & $41 \%$ & $51 \%$ & $29 \%$ \\
\hline
\end{tabular}


Nota-se alguma estabilidade nas suas estimativas. Apenas no ano de 1998 houve um leve aumento; mas, no ano seguinte, as volatilidades voltaram para perto do que eram em 1997. Isso sugere que a estimativa da volatilidade com base em dados passados é bem mais razoável do que o mesmo tipo de estimativa feita para a taxa de retorno.

A amostra do Exemplo 3 contou com 52 observações, sempre dentro do próprio ano. Mas nada obriga a que a amostra tenha esse tamanho; logo, a estimativa da volatilidade pode variar, dependendo do prazo da amostra utilizada. Tomando, por exemplo, o fundo BB Fundo de Ações, mostramos, no Gráfico 1, a evolução da volatilidade histórica baseada em amostra das últimas 20 e 60 semanas. A cada semana é acrescentada uma nova semana e retirada a mais antiga; faz então uma janela sempre com 20 (ou 60) semanas, de onde se calculou a volatilidade expressa em forma anual.

\section{Gráfico 1: Evolução da Volatilidade Anual do Fundo de Ações do BB}

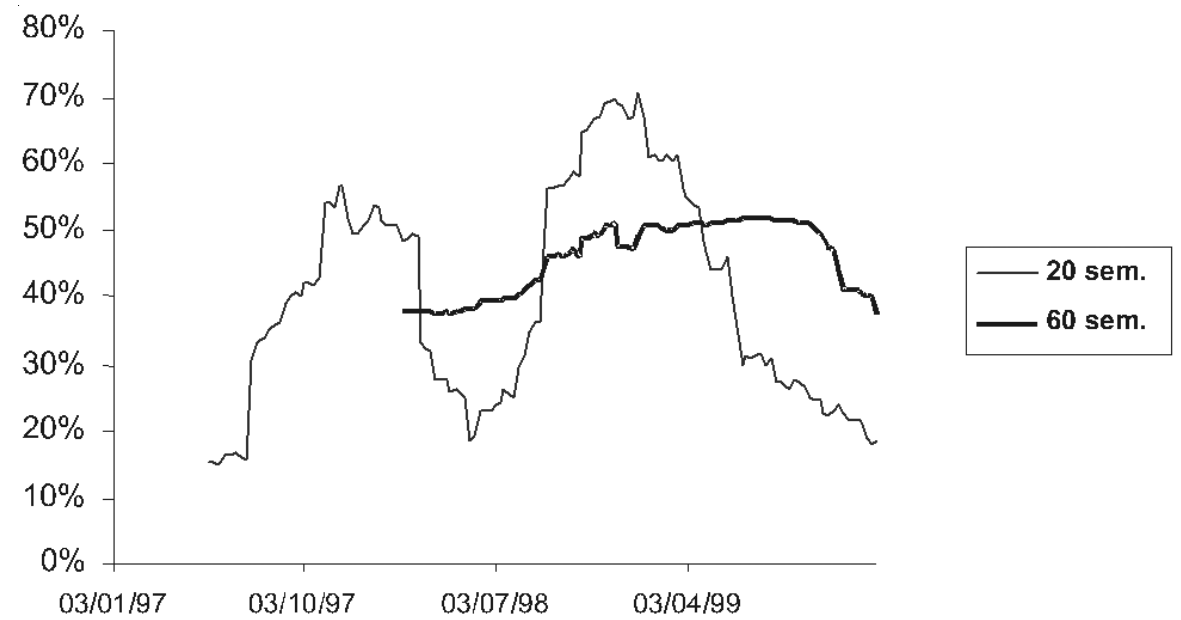

Há grande diferença, em vários momentos, entre as volatilidades com base numa série de 20 e 60 semanas, o que pode levar a diferentes conclusões a respeito do risco de um fundo.

Várias alternativas concorrem para se chegar a diferentes estimativas históricas da volatilidade: tamanho da série histórica dos retornos, periodicidade da taxa de retorno ou procedimento estatístico utilizado. Uma alternativa para se obter a volatilidade, sem depender do passado, é a volatilidade implícita nas opções sobre os ativos que compõem o fundo.

Uma vez determinados os parâmetros da distribuição da taxa de retorno dos 
fundos em estudo, podemos comparar suas relações retorno-risco. Uma referência importante para a avaliação de um fundo é a carteira de mercado ou algum índice que busca representar o mercado, caracterizando-se como benchmark. Esse benchmark é especialmente importante, porque existem fundos oferecidos a custos (taxa de administração e de performance) menores do que a maioria dos outros, que se propõem a apenas acompanhar o benchmark e são conhecidos como de gestão passiva.

Se existe a possibilidade de tomar ou aplicar dinheiro à taxa de juros sem risco, podemos combinar aplicações na carteira de mercado e no título de renda fixa sem risco, de forma a obter qualquer relação retorno-risco. Esse conjunto de combinações gera a chamada linha do mercado de capitais (LMC).

$\mathrm{O}$ retorno de uma carteira que tem $\mathrm{x} \%$ no ativo arriscado e $(1-\mathrm{x} \%)$ no ativo sem risco tem retorno esperado e risco dados por:

$$
\begin{aligned}
& E\left(r_{C}\right) \quad x E\left(r_{A}\right)\left(\begin{array}{ll}
1 & x
\end{array}\right) r_{s r} \\
& \text { C } x \text { A }
\end{aligned}
$$

Quando $x$ é menor do que 1, o investidor estará aplicando na renda fixa sem risco e no ativo arriscado; quando $x$ é maior do que 1 , o investidor estará tomando emprestado para alavancar seu investimento no ativo arriscado. As possíveis combinações de retorno-risco para uma carteira estão no Gráfico 2.

\section{Gráfico 2: Combinações de Retorno e Risco}

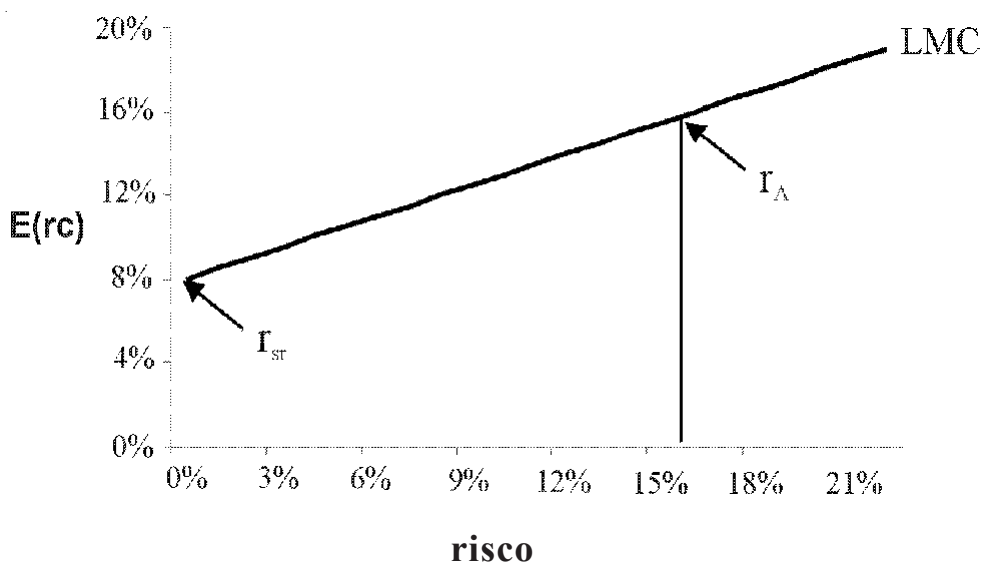


Das Equações [6a] e [6b] temos:

$$
\begin{aligned}
& E\left(r_{C}\right) \quad r_{s r} \quad x \quad E\left(r_{A}\right) \quad r_{s r} \quad \text { e } \quad x \quad C / A \\
& \left.E\left(r_{C}\right) \quad r_{s r} \quad \stackrel{C}{E\left(r_{A}\right)} r_{s r}\right] \\
& \text { A }
\end{aligned}
$$

O retorno esperado das carteiras que combinam o ativo sem risco e o ativo arriscado vem de uma linha reta com intercepto igual a $r_{s r}$ e inclinação igual a:

$$
\frac{E r_{A} \quad r_{s r}}{A}
$$

Esse é o conjunto de possibilidades eficientes que um investidor faceia!

As carteiras de investimento com relação retorno-risco menor do que as contempladas no LMC são facilmente superadas por uma combinação do ativo sem risco e da carteira arriscada que está na LMC. Com esse tipo de combinação, podemos gerar uma carteira com qualquer nível de risco e retorno.

Exemplo 4. Sejam as seguintes condições de mercado:

\begin{tabular}{ccc}
\hline & Retomo & Volatilidade \\
\hline Taxa sem risco & $10 \%$ & $0 \%$ \\
Fundo X & $20 \%$ & $30 \%$ \\
Fundo Y & $25 \%$ & $55 \%$ \\
\hline
\end{tabular}

Com esses valores, pode-se montar uma carteira de valor inicial $\$ 100$, com uma captação de $\$ 50$ e aplicação de $\$ 150$ no fundo $X$, que terá um retorno esperado de $25 \%$ e volatilidade de $45 \%$. Se a captação for de $\$ 83,2$, e a aplicação no fundo X de $\$ 183,2$, o retorno da carteira deve ser de $28,3 \%$, e volatilidade de $55 \%$. Assim, o fundo Y, que proporciona um retorno maior, mas com maior volatilidade, é ineficiente, pois a combinação do fundo X com a aplicação no título sem risco gera uma carteira com mesma volatilidade e retorno esperado maior.

A simples relação retorno-risco não é adequada para selecionar um 
fundo e pode levar a uma decisão $E r_{C} \int_{C}$ errônea, como ilustrado no Exemplo 5 .

Exemplo 5. Sejam dois fundos, $\mathrm{X}$ e Y, com os seguintes retornos e volatilidades esperados:

\begin{tabular}{lccc}
\hline & $\begin{array}{c}\text { Retormo } \\
\text { esperado }\end{array}$ & $\begin{array}{c}\text { Volatilidade } \\
\text { esperada }\end{array}$ & $\begin{array}{c}\text { Retorno } \\
\text { /risco }\end{array}$ \\
\hline Fundo X & $5 \%$ & $10 \%$ & 0,50 \\
Fundo Y & $8 \%$ & $20 \%$ & 0,40 \\
\hline
\end{tabular}

Se a taxa de juros sem risco é de $3 \%$, e um investidor quer um investimento com $10 \%$ de volatilidade, irá selecionar o fundo X se usar a relação retorno/risco como seu indicador para seleção de fundos. Mas é fácil verificar que, se ele investir 50\% no título sem risco e 50\% no fundo $\mathrm{Y}$, a volatilidade de seu investimento será de $10 \%$, e o retorno esperado de $5,5 \%$; portanto, superior ao do fundo X. O indicador correto é o da Equação [6c].

Fundos diferentes costumam ter alta correlação entre si, por estarem sujeitos a variações em fatores macroeconômicos que têm efeito sobre os ativos de todos os fundos. Se um único fator explica parcela importante da variação dos preços dos ativos, podemos explicar a variação do retorno de cada fundo pela sua sensibilidade ao fator (risco sistemático) e a variação restante por componentes específicos do fundo (risco não-sistemático). O fator mais utilizado na análise de performance costuma ser o próprio retorno de mercado, suposto ser um bom representante das variáveis macro e na prática representado por algum índice. Com base nesse modelo, podemos separar o excesso de retorno proporcionado por algum fundo no seu componente sistemático e não-sistemático segundo:

$$
\begin{array}{rlllll}
r_{c} & r_{s r} \quad{ } r_{M} & r_{s r} & e_{C}
\end{array}
$$

O primeiro termo à direita da Equação [7], alfa, mede o excesso de retorno do fundo para dado risco de mercado assumido por ele.

A variância do excesso de retorno do fundo pode ser decomposta no seu risco sistemático e não-sistemático:

$$
\begin{array}{lllll}
2 & 2 & 2 & 2 & e_{C}
\end{array}
$$


O primeiro termo do lado direito é a parcela do risco do fundo explicada pelo risco do mercado (sistemático), e o segundo termo é o risco não explicado pelo mercado. Um fundo passivo que acompanha perfeitamente o retorno do mercado não irá gerar risco diferente do mercado. Já um fundo ativo que procura superar o mercado tem de ter ações diferentes da carteira do mercado, para ter a chance de superar o mercado. O preço pago para se ter essa chance é o de ter uma carteira que eventualmente não acompanha (às vezes supera) o retorno do mercado, cuja possível conseqüência é um risco maior mensurado pelo segundo termo à direita da Equação [8].

Com isso, fazemos uma melhor decomposição e obtemos uma melhor explicação do eventual sucesso ou infortúnio de certo fundo de investimento. Por exemplo, tomando o Ibovespa como retorno de mercado e o CDI como taxa sem risco, explicamos o retorno semanal do fundo BB de ações entre 1997 e 1999 por:

\section{Resumo dos Resultados}

\begin{tabular}{lr}
\hline \multicolumn{2}{c}{ Estatistica de regressão } \\
\hline R-Quadrado & $87 \%$ \\
Observações & 157 \\
\hline
\end{tabular}

\begin{tabular}{lcc}
\hline & $G l$ & $S Q$ \\
\hline Risco sistemático & 1 & 0,4361 \\
Risco não-sistemático & 155 & 0,0635 \\
Risco total & 156 & 0,4996 \\
\hline
\end{tabular}

\begin{tabular}{lrrr}
\hline & Coeficientes & Erro-padrão & Stat t \\
\hline Alfa & $(0,0029)$ & 0,0016 & $(1,81)$ \\
Beta & 0,86 & 0,0263 & 32,62 \\
\hline
\end{tabular}

De acordo com essa amostra de três anos, o fundo BB pode ser tratado como um fundo conservador, por ter um beta menor do que 1 que representa um pequeno risco não-sistemático e retorno ajustado ao risco de mercado negativo (alfa).

O retorno extraordinário proporcionado por certo fundo de investimento pode não ter sido resultado de uma boa gestão ativa, mas simplesmente do seu risco sistemático (quantificado pelo beta). Um fundo com beta menor do que 1 possi- 
velmente terá um retorno superior ao do mercado quando este cair. Se o beta for maior do que 1, o retorno do fundo deve ultrapassar o retorno do mercado, se este subir. Assim, a taxa de retorno não é uma estatística adequada para classificar diferentes fundos, porque, entre outras coisas, não ajusta o retorno para o risco assumido pelo fundo.

Exemplo 6. Com base nos dados do Exemplo 3, calculamos o beta, retorno efetivo e classificamos os fundos.

\section{Quadro 3: Beta, Retorno Efetivo e Classificação dos Dez Maiores Fundos de Ações}

\begin{tabular}{|c|c|c|c|c|c|c|}
\hline & \multicolumn{2}{|c|}{ Beta } & \multicolumn{2}{|c|}{ Retorno efetivo } & \multicolumn{2}{|c|}{ Classificação } \\
\hline & 1997 & 1998 & 1997 & 1997 & 1997 & 1998 \\
\hline BB FUNDO DE AÇÕES & 0,86 & 0,84 & $18 \%$ & $-39 \%$ & 9 & 9 \\
\hline BOSTON AÇÕES & 1,00 & 1,10 & $34 \%$ & $-33 \%$ & 4 & 5 \\
\hline CCF-AÇÕES & 1,07 & 1,11 & $56 \%$ & $-35 \%$ & 1 & 7 \\
\hline CITIAÇÕES & 1,02 & 1,07 & $28 \%$ & $-33 \%$ & 6 & 4 \\
\hline ITAUAÇÕES & 0,72 & 0,66 & $27 \%$ & $-33 \%$ & 7 & 6 \\
\hline UNIBANCO BLUE & 1,00 & 0,92 & $24 \%$ & $-36 \%$ & 8 & 8 \\
\hline IP PARTICIPAÇÕ ES & 0,18 & 0,48 & $-6 \%$ & $-18 \%$ & 10 & 1 \\
\hline BRADESCO CART. LIVRE & 1,04 & 1,11 & $31 \%$ & $-42 \%$ & 5 & 10 \\
\hline UNIBANCO STRATEGY & 0,97 & 0,96 & $45 \%$ & $-32 \%$ & 3 & 3 \\
\hline OPPORTUNITY LÓGICA II - CL & 0,94 & 0,70 & $46 \%$ & $-30 \%$ & 2 & 2 \\
\hline CDI & & & $24 \%$ & $29 \%$ & & \\
\hline Ibovespa & & & $40 \%$ & $-31 \%$ & & \\
\hline
\end{tabular}

Dois fundos que têm sua classificação significativamente alterada são o CCF Ações e o IP Participações. O primeiro tem um beta alto e, como esperado, é um fundo de sucesso em 1997, quando a bolsa sobe. O segundo tem beta baixo e é mais bem-sucedido em 1998, quando a bolsa cai. Tomar esses valores como estimativa para o que vai acontecer com o retorno é questionável ${ }^{(6)}$.

Uma questão prática interessante é qual a taxa de retorno sem risco relevante no Brasil. Aqui, os praticantes se dividem entre taxa de juros da poupança e a do CDI. Claramente, a taxa do CDI é maior que a da poupança, conforme atesta o Quadro 4, embora não seja tão claro quem tem menos risco. Nos livros de finanças, toma-se como taxa sem risco a taxa dos títulos do Governo Federal, já que ele tem poder de emitir moeda e assim pagar qualquer dívida na moeda local; logo, os títulos por ele emitidos têm o menor risco. Comparando-se a taxa de um dia para financiamento de títulos públicos federais - chamada de taxa Selic, a taxa diária do CDI - que se refere a títulos privados de alta qualidade de crédito 
e a taxa da poupança, verifica-se que a taxa Selic é muito próxima da taxa do CDI, e ambas são superiores à taxa da poupança. Na verdade, a taxa da poupança é sempre menor, por se tratar de um mercado cativo, que atinge uma grande massa de investidores e tem custo operacional alto. Para fins de avaliação de fundos, a taxa de juros sem risco mais apropriada é a taxa dos títulos públicos federais (taxa Selic), que é muito próxima da taxa do CDI. Como a taxa Selic não está facilmente disponível, tomamos a taxa do CDI como taxa sem risco.

\section{Quadro 4: Evolução das Taxas de Poupança, Selic e CDI}

\begin{tabular}{|c|c|c|c|}
\hline \multicolumn{4}{|c|}{ Taxa efetiva mensal \% } \\
\hline & Poupança & Selic & CDI \\
\hline Janeiro de 1997 & 1,25 & 1,73 & 1,74 \\
\hline Fev. & 1,16 & 1,67 & 1,66 \\
\hline Mar. & 1,13 & 1,64 & 1,62 \\
\hline Abr. & 1,12 & 1,66 & 1,66 \\
\hline Mai. & 1,14 & 1,58 & 1,58 \\
\hline Jun. & 1,16 & 1,61 & 1,59 \\
\hline Jul. & 1,16 & 1,60 & 1,61 \\
\hline Ago. & 1,13 & 1,59 & 1,58 \\
\hline Set. & 1,15 & 1,59 & 1,58 \\
\hline Out. & 1,16 & 1,67 & 1,68 \\
\hline Nov. & 2,04 & 3,04 & 2,98 \\
\hline Dez. & 1,82 & 2,97 & 2,91 \\
\hline Janeiro de 1998 & 1,65 & 2,67 & 2,67 \\
\hline Fev. & 0,95 & 2,13 & 2,11 \\
\hline Mar. & 1,40 & 2,20 & 2,18 \\
\hline Abr. & 0,97 & 1,71 & 1,69 \\
\hline Mai. & 0,96 & 1,63 & 1,63 \\
\hline Jun. & 0,99 & 1,60 & 1,60 \\
\hline Jul. & 1,05 & 1,70 & 1,69 \\
\hline Ago. & 0,88 & 1,48 & 1,47 \\
\hline Set. & 0,95 & 2,49 & 2,49 \\
\hline Out. & 1,39 & 2,94 & 2,93 \\
\hline Média & 1,21 & 1,95 & 1,94 \\
\hline
\end{tabular}

Tomando a esperança da Equação [7], chega-se ao famoso modelo CAPM, que postula uma relação de equilíbrio entre o retorno esperado de cada ativo e o retorno esperado para o mercado. Nas aplicações do CAPM, trata-se exclusivamente dos retornos esperados e do retorno de todo o mercado: 


$$
\begin{array}{llllll}
E & r_{C} & r_{s r} & { }_{C} & { }_{C} & r_{s r}
\end{array}
$$

Em equilíbrio o CAPM postula que o alfa de qualquer ativo ou fundo deve ser igual a zero.

\section{Í ÍNICE de SHARPE}

Extremamente celebrado entre acadêmicos e praticantes do mercado financeiro, o IS tem sido amplamente utilizado na avaliação de fundos de investimento. Formulado por Sharpe (1966), o IS se encaixa na teoria de seleção de carteira, mais especificamente no modelo CAPM, apontando as carteiras ótimas na LMC. De acordo com o CAPM, nenhuma carteira pode ter um IS maior do que o definido pela carteira de mercado. Carteiras com IS menor devem ser desprezadas.

O IS é a própria inclinação da LMC, definido de [6c], como:

$$
I S \frac{E r_{C} r_{s r}}{C}
$$

onde $r_{s r}$ é a taxa de juros sem risco; $E\left(r_{c}\right)$ é o retorno esperado do fundo; $\sigma_{c}$ é a volatilidade do fundo.

Tendo determinado quais as carteiras ótimas, o investidor deve apenas selecionar aquela que proporciona a relação risco-retorno mais adequada às suas demandas pessoais.

Diversos cuidados devem ser tomados ao se aplicar o IS na seleção ou classificação de investimentos. O primeiro deles vem do fato de o cálculo do IS não incorporar informação sobre a correlação entre os ativos; portanto, o IS perde importância, quando se quer adicionar um ativo (ou carteira) com risco a uma carteira que já tenha ativos arriscados. Quanto maior a correlação entre o ativo que está sendo avaliado e a carteira corrente, maior a importância do IS como indicador para a seleção de um investimento. Se a correlação é muito baixa ou negativa, um ativo com pequeno IS pode tornar ainda maior o IS final de toda a carteira. Um investidor que não tem investimentos com risco deve simplesmente selecionar aquele com maior IS.

Por exemplo, se o investidor já possui investimentos em fundo e procura adicio- 
nar um novo fundo à sua carteira de investimentos, a seleção com base no IS perde importância, porque o efeito da correlação entre o novo fundo e os fundos já existentes na carteira não está sendo considerado pelo IS. O Exemplo 7 ilustra o efeito da correlação sobre o problema da seleção de fundos com base no IS.

Exemplo 7. Seja uma carteira que contenha $50 \%$ de seus investimentos em um título sem risco e $50 \%$ no fundo $\mathrm{X}$ arriscado. O investidor quer aumentar o retorno trocando $20 \%$ do seu investimento no título sem risco pelo fundo $\mathrm{Y}$ ou $\mathrm{Z}$.

\begin{tabular}{lccc}
\hline & \multicolumn{3}{c}{ Dados de mercado } \\
& Retomo (\%) & Volatilidade (\%) & IS \\
\hline Taxa sem risco & 10 & 0 & \\
Fundo X & 21 & 32 & 0,34 \\
Fundo Y & 20 & 26 & 0,38 \\
Fundo Z & 22 & 35 & 0,34 \\
\hline Correlação (X, Y) & & 50 & \\
Correlação (X, Z) & & 20 & \\
Correlação (Y, Z) & & 70 & \\
\hline
\end{tabular}

$\mathrm{O}$ fundo $\mathrm{Y}$ tem um IS maior do que o do fundo $\mathrm{Z}$, mas a aquisição do fundo $\mathrm{Z}$ leva a uma melhor relação risco-retorno final. Isso é devido à menor correlação entre o fundo $\mathrm{Z}$ e o fundo $X$ que já está na carteira, que faz com que a volatilidade da carteira diminua, conforme atesta o quadro abaixo.

\begin{tabular}{lccc}
\hline & Carteiras & \multicolumn{2}{c}{ Altemativa de carteira } \\
& Corrente (\%) & $\mathbf{1}(\boldsymbol{\%})$ & $\mathbf{2}(\boldsymbol{\%})$ \\
\hline Taxa sem risco & 50 & 30 & 30 \\
Fundo X & 50 & 50 & 50 \\
Fundo Y & 0 & 0 & 20 \\
Fundo Z & 0 & 20 & 0 \\
\hline & & & \\
Retorno & 15,5 & 17,9 & 17,5 \\
Volatilidade & 16,0 & 18,7 & 19,1 \\
& & & \\
\hline
\end{tabular}

Com a adição do fundo $\mathrm{Z}$ (primeira alternativa), que tem menor IS, a carteira fica com um retorno esperado de $17,9 \%$ e $18,7 \%$ de volatilidade, contra um retor- 
no menor e volatilidade maior se tivesse adicionado o fundo Y (segunda alternativa).

Um segundo cuidado na utilização do IS decorre de este ser baseado em retorno e risco esperados (ex-ante) e retorno não-realizado (ex-post). Dada a dificuldade em se obterem valores esperados, muitos praticantes utilizam estatísticas passadas para avaliar o IS. O resultado pode ser muito ruim, levando eventualmente a um IS negativo, quando a bolsa cai. O IS negativo não tem sentido num modelo de mercado, pois o investidor sempre tem a opção de investir na taxa sem risco.

Se a estimativa do IS for feita com base em dados históricos, uma dificuldade prática é determinar qual o tamanho de série a ser utilizado para o cálculo do retorno e risco esperado. Uma saída para essa dificuldade é tomar o número de observações que proporcionam certo nível de confiança.

Para se calcular o IS ex-post, devemos, a cada período, obter o excesso de retorno do fundo em relação à taxa sem risco; tomamos a média e o desviopadrão do excesso de retorno, e a razão desses é o IS ex-post. Ele indica o excesso de retorno histórico médio por unidade de variabilidade histórica do excesso, e é dado por:

$$
\bar{r}_{c} \frac{1}{T j}{ }_{j}^{T} r_{c, j} ; \bar{r}_{s r} \frac{1}{T j}{ }_{j}^{T} r_{s r, j} ; C \sqrt{\frac{T_{j 1} r_{c, j}}{T_{1}} \bar{r}_{c}}
$$

onde $r_{c, t}$ é o retorno do fundo e $r_{s r, t}$ é o retorno sem risco.

Por fim, o IS é obtido por:

$$
\hat{I S} \frac{\bar{r}_{c} \bar{r}_{s r}}{C}
$$

O IS é uma estatística que depende do período sobre o qual foi calculada; uma vez calculada para certo prazo, pode $\operatorname{ser}^{(7)}$ transformada para prazo diferente, de acordo com a seguinte aproximação:

$$
\text { IS } S_{\text {anual }} \sqrt{252} I S_{\text {diário }}
$$


Exemplo 8. Tomando os dados do Exemplo 3, para o fundo de ações do BB, calculamos o IS anualizado, com base numa janela dos retornos das últimas $20 \mathrm{e}$ 60 semanas. A taxa de juros sem risco utilizada foi a do CDI. O resultado está no Gráfico 3.

\section{Gráfico 3: IS para o Fundo de Ações do BB, com base numa Amostra Variável das Últimas 20 e 60 Semanas}

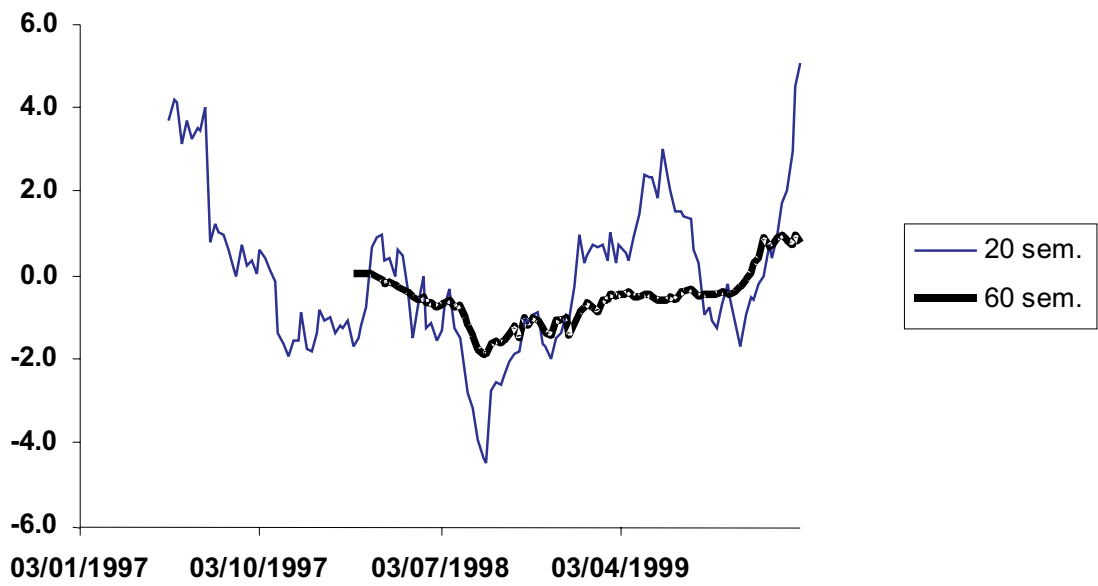

É interessante observar, no Gráfico 3, que o IS fica por várias vezes negativo, além de flutuar exageradamente. O IS fica negativo, porque o retorno esperado para carteira é menor do que a taxa de juros sem risco, que tem pouco sentido econômico, e ocorre especialmente quando o retorno esperado é estimado com base na série histórica.

Outro problema que também surge na utilização do IS é sua aplicação a ativos que têm volatilidade muito baixa, como são os fundos de renda fixa. O IS obtido fica enorme, devido ao baixo valor do denominador da fórmula.

Sharpe (1994) dá uma interpretação muito interessante ao IS. Ele trata esse índice como um instrumento de avaliação de uma estratégia de investimento zero, que corresponde ao retorno da arbitragem entre algum benchmark e o fundo que está sendo avaliado. Então, o IS tradicional pode ser interpretado como uma arbitragem entre a taxa de juros sem risco e o fundo que está sendo avaliado. Pode-se usar algum índice de mercado, como o Ibovespa, no lugar da taxa de juros sem risco. Nesse caso, o IS está mostrando o resultado de uma arbitragem entre uma posição vendida no índice do mercado e uma comprada no fundo. O IS 
modificado dessa forma foi chamado por Brito (1996) de índice de Sharpe generalizado (ISG).

Se o benchmark adotado para o cálculo desse índice for igual ao estilo ${ }^{(8)}$ adotado pelo gestor, o resultado apresentado pelo ISG é o excesso de retorno devido à capacidade do gestor em selecionar bem os títulos que compõem a carteira de seu fundo.

\section{Outras Medidas}

O modelo de um fator representado pela Equação [7] permite obter vários outros indicadores de performance importantes que vão (1) agregar informação sobre a contribuição de um gestor para a performance do seu fundo; (2) gerar classificações diferentes para os fundos disponíveis; e (3) ser apropriado para um cenário específico de investimento. A principal diferença entre esses indicadores vem da medida de risco utilizada: ora é o risco total, ora o risco de mercado ou risco não-sistemático apenas.

O alfa da Equação [7] mede o excesso de retorno obtido pelo fundo após ajuste pelo risco sistemático (dado pelo beta vezes o excesso de retorno do mercado). Com base na estimativa da Equação [7], temos

$$
\hat{c}_{C} \bar{r}_{C} \quad \bar{r}_{s r} \quad \underbrace{\hat{C}_{C} \bar{r}_{M} \quad \bar{r}_{s r}}_{\text {retorno devido ao riscosistemático }}
$$

que é o indicador conhecido como índice de Jensen ou simplesmente alfa. Esse indicador desqualifica gestores que são bem-sucedidos na alta (baixa) do mercado apenas por terem um beta maior (menor) do que 1 .

Um gestor ativo bem-sucedido deve mostrar um alfa positivo. Mas, para conseguir superar o mercado, também deve adquirir uma carteira diferente da carteira do mercado (ou do índice que representa o mercado), e o preço por isso é uma menor diversificação, que estará contida no termo de erro da Equação [7]. Então, dividindo-se o alfa da carteira pelo seu risco não-sistemático, tem-se o índice de valor adicionado (IVA). Esse indicador mostra quanto determinado gestor pôde adicionar de valor a uma carteira com vários outros fundos, pelo fato de assumir um risco diferente do risco de mercado. 


$$
\stackrel{\wedge}{\stackrel{\wedge}{ } \stackrel{ }{\wedge} \frac{C}{e_{C}}}
$$

Outro indicador extraído da Equação [7] é o índice de Treynor (IT), que mede o excesso de retorno por unidade de risco sistemático $^{(9)}$, em vez do risco total, como no IS.

$$
\hat{I T} \frac{\bar{r}_{C} \bar{r}_{s r}}{\hat{C}_{C}}
$$

O valor do IS é de difícil interpretação, mas outro indicador, chamado $M^{2}$ e diretamente relacionado ao IS, também ajusta o retorno ao risco total (medido pela volatilidade total do mercado). Foi criado recentemente (1997) por Leah Modigliani e seu avô Franco Modigliani, ganhador do Prêmio Nobel de Economia. É uma medida de performance que mostra o diferencial de retorno do fundo e do mercado após ajustar o retorno do fundo ao risco de mercado, como se ambos tivessem a mesma volatilidade. Para se obter o $M^{2}$, deve-se primeiramente obter o retorno ajustado, que é igual ao retorno original da carteira alavancada de forma a ter o mesmo risco do mercado. Para ter o mesmo risco do mercado, a carteira ajustada deve ter $x \%$ investido na carteira original, tal que:

$$
\begin{array}{lllll}
M & x \% & C & x \% & \frac{M}{C}
\end{array}
$$

Com essa quantidade investida na carteira original, o retorno da carteira ajustada é de:

$$
r_{c a} \quad \frac{M}{C} r_{C} \quad 1 \quad \frac{M}{C} r_{s r}
$$

O $M^{2}$ é a diferença entre o retorno da carteira ajustada e o retorno de mercado.

$$
\begin{array}{lll}
M^{2} & r_{c a} & r_{M}
\end{array}
$$

Esse indicador ajusta a volatilidade do fundo à volatilidade do mercado, de 
modo a verificar qual teria sido o retorno, se o fundo tivesse o mesmo nível de risco do mercado $\left(\sigma_{c}=\sigma_{M}\right)$. Por construção, a volatilidade da carteira ajustada fica igual à do mercado:

\begin{tabular}{|c|c|c|c|c|c|c|c|}
\hline \multirow{3}{*}{$\begin{array}{l}2 \\
c a\end{array}$} & \multicolumn{2}{|c|}{2} & \multicolumn{3}{|c|}{2} & & \\
\hline & $M$ & 2 & 1 & $M$ & 2 & & \\
\hline & & $c$ & & & $s r$ & $c a$ & $M$ \\
\hline
\end{tabular}

Para efeito de comparação, transformamos todos esses indicadores nos seus equivalentes anuais, usando o mesmo critério do IS em [11a]. Então, novamente desprezamos o efeito da composição dos retornos e temos para a amostra semanal do Exemplo 9:

$$
\wedge_{\text {anual }} 52 \hat{\imath}_{\text {semanal }} ; \hat{I V A} \sqrt{52} \frac{\hat{C}_{C}}{{ }_{C}} ; \hat{I T} 52 \frac{\bar{r}_{C} \bar{r}_{s r}}{\hat{\imath}_{C}} \text {. }
$$

Exemplo 9. Tomando os dados semanais do Exemplo 3, calculamos os indicadores acima e apresentamos seus valores no Quadro 5.

\section{Quadro 5: Diversas Medidas de Performance para os Dez Maiores Fundos de Ações Brasileiros - Amostra}

\begin{tabular}{lccccc}
\hline \multicolumn{1}{c}{ Fundo } & IS & IT & Alfa & IVA & $\mathbf{M}^{\mathbf{2}}$ \\
\hline BB FUNDO DE AÇÕES & $(0,02)$ & $-1 \%$ & $-15 \%$ & $-104 \%$ & $-23,9 \%$ \\
BOSTON AÇÕES & 0,28 & $13 \%$ & $-4 \%$ & $-32 \%$ & $-9,9 \%$ \\
CCF-AÇÕES & 0,37 & $17 \%$ & $1 \%$ & $5 \%$ & $-4,0 \%$ \\
CITIAÇÕES & 0,32 & $15 \%$ & $-2 \%$ & $-16 \%$ & $-3,2 \%$ \\
ITAUAÇÕES & 0,32 & $16 \%$ & $-1 \%$ & $-3 \%$ & $8,9 \%$ \\
UNIBANCO BLUE & 0,16 & $8 \%$ & $-9 \%$ & $-61 \%$ & $-14,7 \%$ \\
P PARTICIPAÇÕES & 0,32 & $24 \%$ & $26 \%$ & $114 \%$ & $25,1 \%$ \\
BRADESCO CART. LIVRE & 0,15 & $7 \%$ & $-10 \%$ & $-73 \%$ & $-16,9 \%$ \\
UNIBANCO STRATEGY & 0,31 & $14 \%$ & $-2 \%$ & $-17 \%$ & $-7,8 \%$ \\
OPPORTUNITY LÓGICA II - CL & 0,72 & $40 \%$ & $21 \%$ & $69 \%$ & $41,9 \%$ \\
\hline
\end{tabular}

Observando-se o Quadro 5, verifica-se que o fundo BB tem uma performance inferior por qualquer indicador. Apenas o IP Participações e o Opportunity apresentam performance positiva segundo quaisquer desses indicadores. Ajustandose a volatilidade do fundo à volatilidade do mercado, o que é feito pelo $M^{2}$, o fundo Itauações também mostra retorno positivo. 
Uma estratégia de negócio interessante para um fundo de fundos, que salta aos olhos após se calcular o $M^{2}$, é a combinação de fundos com relação retorno-risco superior e volatilidades diferenciadas. Como existem entraves legais à alavancagem de fundos, pode-se usar um derivativo (contrato futuro de Ibovespa) para, na prática, capturar o benefício de um $M^{2}$ superior.

Exemplo 10. Seja um fundo X com $M^{2}$ superior e baixa volatilidade corrente; combinamos esse fundo com uma carteira de mercado, com o objetivo de obter uma carteira final com volatilidade igual à do mercado e retorno superior.

\begin{tabular}{cccc}
\hline & Volatilidade & Retomo & $\begin{array}{c}\text { Composição } \\
\text { da carteira }\end{array}$ \\
\hline Fundo X & $35,0 \%$ & $45,0 \%$ & $100,0 \%$ \\
Mercado & $40,0 \%$ & $45,0 \%$ & $12,5 \%$ \\
Título sem risco & $0,0 \%$ & $20,0 \%$ & $-12,5 \%$ \\
\hline
\end{tabular}

Essa carteira está $100 \%$ comprada no fundo X e tem mais $12,5 \%$ aplicados no mercado (o que pode ser feito com a compra de um derivativo ${ }^{(10)}$ ) com uma captação também de $12,5 \%$ a taxa sem risco (que é a taxa implícita no derivativo).

\begin{tabular}{rcc}
\hline & Volatilidade & Retomo \\
\hline Carteira final & $40,0 \%$ & $48,1 \%$ \\
Correlação (Fundo X, mercado) & $80,0 \%$ & \\
\hline
\end{tabular}

Com tal combinação de ativos chega-se a uma carteira com volatilidade igual à do mercado $(40 \%)$ e retorno superior $(48,1 \%)$.

\section{Qual o Melhor Fundo?}

Essa é a pergunta a que se quer responder com os indicadores apresentados até aqui. E a resposta depende também do investidor e de sua carteira corrente. Se as preferências dos investidores são representadas pela média e volatilidade, os indicadores apresentados permitem um critério bastante simples de seleção. O objetivo final do investidor é que sua carteira total, que é a soma da carteira corrente mais qualquer novo investimento, tenha o máximo IS. Então os indica- 
dores permitirão avaliar o impacto de qualquer novo fundo sobre a carteira do investidor.

O indicador apropriado para a seleção de carteira depende da importância da carteira para o investidor e são três as possibilidades. A primeira trata do investidor que não tem investimento com risco e procura selecionar alguma carteira para ser seu único investimento com risco. Esse é o caso mais simples, e a carteira com maior IS é a mais adequada. Pela amostra apresentada no Exemplo 9, o fundo mais indicado, nesse caso, é o Opportunity. Note que o indicador $M^{2}$ cumpre um papel semelhante ao IS, pois ajusta o retorno à volatilidade e, no Exemplo 9, aponta o mesmo fundo como sendo o melhor.

O segundo caso trata de quando o investidor já possui a carteira de mercado e quer adicionar outro investimento arriscado. Nesse caso, o indicador apropriado é o IVA, que mede, por meio do alfa, quanto retorno pode ser adicionado em troca da adição de um risco não-sistemático dado por $\sigma\left(e_{c}\right)$. O IVA entra como uma medida de custo-benefício da inclusão de um novo fundo. Então, de acordo com o resultado do Exemplo 9, o melhor fundo continua sendo o do Opportunity.

$\mathrm{O}$ terceiro caso ocorre quando o investidor tem diversos fundos e quer avaliar a performance deles para poder realocar seus investimentos e aumentar a performance total. Intuitivamente, nota-se que o risco não-sistemático fica desprezível, já que vários fundos juntos geram uma diversificação suficiente para eliminar esse componente do risco. Assim, o componente do risco total mais importante é o do risco sistemático.

Um indicador que mostra o retorno extraordinário de um fundo é o seu alfa; com base nele, pode-se ter uma idéia da contribuição adicional de certo fundo à carteira total. Mas o retorno extraordinário dado pelo alfa pode ser conseqüência da assunção de um risco sistemático maior (maior beta). Nesse caso, a combinação de uma posição alavancada em um fundo com menor alfa e menor beta e uma posição vendida no título sem risco pode gerar um investimento com um alfa ainda maior. O indicador IT é a medida apropriada para se avaliar a adição de um fundo a uma carteira com vários fundos, porque divide o excesso de retorno de cada fundo pelo seu risco sistemático apenas, e essa é a medida de risco relevante, pois o componente não-sistemático do risco irá desaparecer quando os fundos se combinarem. No caso dos fundos do Quadro 5, o IT do fundo Opportunity também é o maior. Devido ao enorme retorno acumulado desse fundo, todos os indicadores o apontam como o melhor.

Embora esses indicadores destaquem aspectos diferentes de cada fundo, geram quase a mesma classificação (ou ranking), como atesta o Quadro 6. A significância estatística desses indicadores é tanto maior quanto maior o prazo da 
análise, mas não é difícil mostrar que, para se obter algo aceitável, são necessários muitos anos de observações, o que inviabiliza qualquer apuração estatisticamente mais forte. Um arrazoado muito interessante sobre seleção de um fundo de investimento pode ser visto no capítulo 24 do livro de Bodie, Kane e Marcus (1996).

Quadro 6: Classificação dos Fundos segundo Diversos Indicadores, Usando o Ibovespa como Índice de Mercado

\begin{tabular}{lccccc}
\hline \multicolumn{1}{c}{ Fundo } & IS & IT & Alfa & IVA & $\mathbf{M}^{\mathbf{2}}$ \\
\hline BB FUNDO DE AÇÕES & 10 & 10 & 10 & 10 & 10 \\
BOSTON AÇÕES & 7 & 7 & 7 & 7 & 7 \\
CCF-AÇÕES & 2 & 3 & 3 & 3 & 5 \\
CITIAÇÕES & 5 & 5 & 5 & 5 & 4 \\
ITAUAÇÕES & 4 & 4 & 4 & 4 & 3 \\
UNIBANCO BLUE & 8 & 8 & 8 & 8 & 8 \\
IP PARTICIPAÇÕES & 3 & 2 & 1 & 1 & 2 \\
BRADESCO CART. LIVRE & 9 & 9 & 9 & 9 & 9 \\
UNIBANCO STRATEGY & 6 & 6 & 6 & 6 & 6 \\
OPPORTUNITY LÓGICA II - CL & 1 & 1 & 2 & 2 & 1 \\
\hline
\end{tabular}

Uma questão importante no cálculo desses indicadores é a escolha do índice de mercado, e a pergunta, conhecida como crítica de Roll, é qual (se for possível obtê-la) a carteira de mercado. Na avaliação de fundos, podemos gerar diferentes classificações, dependendo do índice de mercado utilizado. Esse problema é discutido em Dybvig e Ross (1985). Tomamos, para efeito de comparação, outro representante da carteira de mercado, o IBX, em vez do Ibovespa. A nova classificação dos fundos está no Quadro 7.

Quadro 7: Classificação dos Fundos segundo Diversos Indicadores, Usando o IBX como Índice de Mercado

\begin{tabular}{llllll}
\hline \multicolumn{1}{c}{ Fundo } & IS & IT & Alfa & IVA & $\mathbf{M}^{\mathbf{2}}$ \\
\hline BB FUNDO DE AÇÕES & 10 & 10 & 10 & 10 & 10 \\
BOSTON AÇÕES & 7 & 7 & 7 & 3 & 7 \\
CCF-AÇÕES & 2 & 3 & 2 & 2 & 5 \\
CITIAÇÕES & 5 & 5 & 4 & 1 & 4 \\
ITAUAÇÕES & 4 & 4 & 5 & 5 & 3 \\
UNIBANCO BLUE & 8 & 8 & 8 & 7 & 8 \\
IP PARTICIPAÇÕES & 3 & 2 & 3 & 9 & 2 \\
BRADESCO CART. LIVRE & 9 & 9 & 9 & 8 & 9 \\
UNIBANCO STRATEGY & 6 & 6 & 6 & 4 & 6 \\
OPPORTUNITY LÓGICA II - CL & 1 & 1 & 1 & 6 & 1 \\
\hline
\end{tabular}


A principal surpresa é a classificação dos fundos pelo IVA, que muito se altera, especialmente com os fundos Opportunity e IP Participações. Isso é devido à grande volatilidade do erro não-sistemático (pouca diversificação) que eles apresentam, quando tomamos o IBX como índice de mercado. $\mathrm{O}$ alfa de todos os fundos melhora significativamente, colocando-os em situação muito melhor.

Em todos os exemplos acima, tomamos os indicadores calculados com base em séries históricas relativamente curtas (três anos) e um conjunto pequeno de fundos. Mostramos apenas um exemplo de como selecionar fundos, não se tratando de um estudo detalhado para determinar qual o melhor fundo de ações. Na prática, deve-se fazer uma avaliação mais apurada dos indicadores, especialmente tentar corrigir erros provenientes do cálculo com base na série histórica e avaliar o comportamento de cada fundo em períodos maiores. O universo também deve ser ampliado, para conter o maior número possível de fundos abertos aos investidores.

\section{TRACKING ERROR}

No caso dos fundos passivos, a principal preocupação dos investidores é saber se o fundo está acompanhando bem o benchmark ou índice que ele se propõe a reproduzir. Um indicador de performance de fundos de investimentos passivos que mede como o fundo acompanha o índice é o tracking error (TE).

$$
T E=\text { desvio }- \text { padrão }\left\{\left(\mathrm{r}_{\mathrm{c}}-\mathrm{r}_{\mathrm{b}}\right)\right\}
$$

onde $r_{b}$ é retorno do benchmark.

Alguns fundos quase-passivos se propõem a superar o índice sem se distanciar sobremaneira dele. São esses os fundos conhecidos como enhanced index strategies. São estratégias de negócios que procuram maximizar o retorno sujeito a minimização de um tracking error preestabelecido. É uma gestão ativa com risco controlado.

Sua função objetivo é:

$$
\begin{aligned}
& \text { Max } r_{C} \\
& \text { sujeito a } \operatorname{var}\left(r_{C} \quad r_{b}\right) \quad \overline{T E} .
\end{aligned}
$$


O tracking error é a diferença no retorno da carteira e do seu benchmark. Os gestores se preocupam com o tracking error, porque se eles tiverem comportamento pior do que seu benchmark, menos investidores irão procurar seus serviços, e eles podem perder o emprego.

Exemplo 11. Tomamos uma amostra de 1998 e 1999 dos dez maiores fundos passivos em Ibovespa oferecidos no mercado brasileiro.

Quadro 8: Retorno Total, Tracking Error (Anualizado) e Excesso de Retorno sobre o Benchmark dos Dez Maiores Fundos de Gestão Passiva Oferecidos no Mercado Brasileiro

\begin{tabular}{|c|c|c|c|c|c|c|}
\hline & \multicolumn{2}{|c|}{ Retorno } & \multicolumn{2}{|c|}{ Tracking error } & \multicolumn{2}{|c|}{$\begin{array}{l}\text { Excesso s/ } \\
\text { benchmark }\end{array}$} \\
\hline & 1998 & 1999 & 1998 & 1999 & 1998 & 1999 \\
\hline ALFA ÍNDICE II FIC FTVM & $-19 \%$ & $89 \%$ & $17 \%$ & $14 \%$ & $12 \%$ & $-65 \%$ \\
\hline CAIXA AÇÕES & $-36 \%$ & $136 \%$ & $13 \%$ & $16 \%$ & $-6 \%$ & $-18 \%$ \\
\hline BB AÇÕES ÍNDICE & $-35 \%$ & $133 \%$ & $18 \%$ & $17 \%$ & $-5 \%$ & $-21 \%$ \\
\hline HEDGING-GRIFFO FCL II & $-34 \%$ & $148 \%$ & $9 \%$ & $17 \%$ & $-3 \%$ & $-6 \%$ \\
\hline HSBC AÇÕES ÍNDICES & $-32 \%$ & $136 \%$ & $11 \%$ & $16 \%$ & $-1 \%$ & $-18 \%$ \\
\hline PERSONNALITÉ MARCHÉ FICFIA & $-32 \%$ & $136 \%$ & $12 \%$ & $16 \%$ & $-2 \%$ & $-17 \%$ \\
\hline ITAÚ ÍNDICE AÇÕES FICFIA & $-33 \%$ & $143 \%$ & $12 \%$ & $17 \%$ & $-2 \%$ & $-11 \%$ \\
\hline SAFRA INDICIAL & $-31 \%$ & $137 \%$ & $14 \%$ & $16 \%$ & $-1 \%$ & $-17 \%$ \\
\hline BRADESCO LIVRE & $-42 \%$ & $127 \%$ & $15 \%$ & $17 \%$ & $-11 \%$ & $-27 \%$ \\
\hline CCF TOP & $-33 \%$ & $144 \%$ & $10 \%$ & $16 \%$ & $-2 \%$ & $-10 \%$ \\
\hline CDI & $29 \%$ & $25 \%$ & & & & \\
\hline Ibovespa & $-31 \%$ & $154 \%$ & & & & \\
\hline
\end{tabular}

O destaque é o fundo Hedging-Griffo FCL II, que tem um dos menores TE e ao mesmo tempo perde muito pouco valor em relação ao índice. É natural que o excesso de retorno seja negativo, pois os retornos são líquidos de todos os custos (exceto impostos). Interessante observar o fundo Alfa Índice, que apresenta um TE próximo aos outros fundos, mas excesso de retorno extremamente diferenciado.

\section{MARKet TIMING}

Com base em um procedimento desenvolvido por Treynor e Mazuy (1966), pode-se avaliar a qualidade do gestor quanto à sua capacidade de acertar os movimentos de curto prazo do mercado. Essa análise é conhecida como market timing (MT). Os indicadores de performance apresentados nas seções anterio- 
res são apropriados para carteiras que não aumentam ou diminuem muito sua exposição ao risco de mercado. Quando se permite, como é na prática, que o gestor varie sua exposição ao risco de mercado, o MT captura o sucesso obtido com essa movimentação.

Em um mercado de alta, o gestor que acerta o movimento deve aumentar sua exposição ao ativo com risco antes da sua alta. Isso deve aumentar o beta da carteira em períodos de alta de preço do ativo arriscado.

$$
\begin{array}{rlllllll}
r_{C} & r_{s r} & r_{M} & r_{s r} & r_{M}{ }^{2} \quad{ }_{C}
\end{array}
$$

O resultado pode ser verificado por meio de uma relação não-linear entre o excesso de retorno da carteira e o excesso de retorno do mercado, como desenhado no Gráfico 4. Isso pode ser quantificado pela equação abaixo, onde o sinal e a significância do seu coeficiente gama $(\gamma)$ irão determinar se o gestor acerta o movimento do mercado.

\section{Gráfico 4: Interpretação Gráfica do MT}

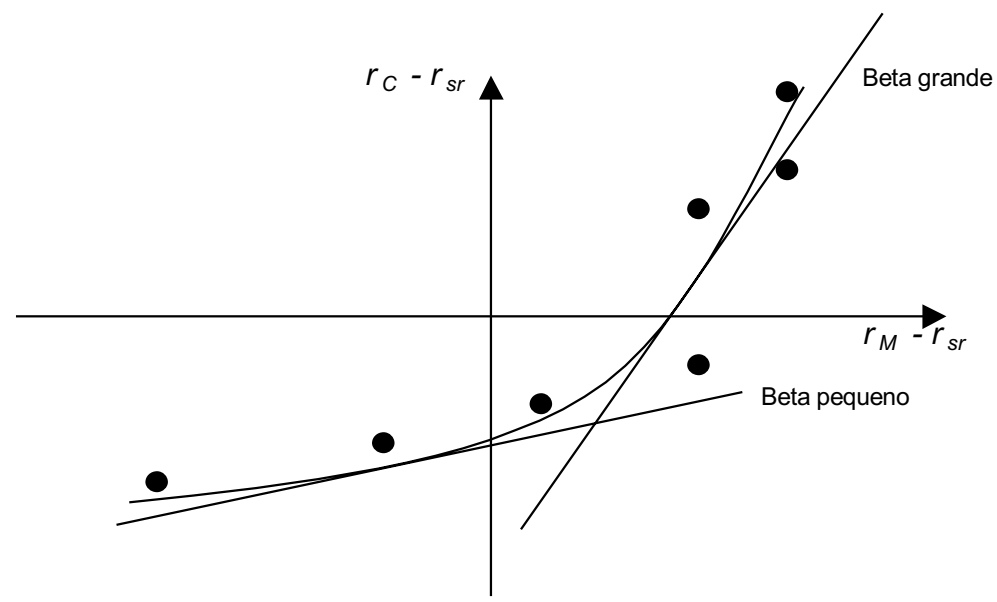

Obs.: o beta (inclinação) da carteira aumenta, quando o mercado está em alta, e diminui, quando o mercado está em baixa.

No Exemplo 12, calculamos os parâmetros da Equação [16] para nossa amostra de fundos de ações. O resultado que se obtém para as outras variáveis, como o alfa e o beta, é diferente do que foi obtido da Equação [7]. Costuma-se vincular 
essas letras gregas à explicação do excesso de retorno obtido pelo fundo: o gama $(\gamma)$ contabiliza o que foi ganho a título de timing; o alfa $(\alpha)$, o que foi ganho pela capacidade do gestor selecionar os títulos (seletividade) que compõem a sua carteira; e o beta $(\beta)$, o risco sistemático (agressividade) assumido. Uma interessante discussão sobre como avaliar a contribuição de um gestor, em termos de timing e seletividade, está em Admati et al. (1986).

Exemplo 12. Novamente tomando os dados dos dez maiores fundos de ações do Exemplo 3, decompomos o retorno dos fundos nas suas variáveis alfa (seletividade), beta (agressividade) e gama (timing).

Quadro 9: Os Parâmetros da Equação [16] Estimados para os Dez Maiores Fundos de Ações

\begin{tabular}{|c|c|c|c|}
\hline & Alfa & Beta & Gama \\
\hline \multirow[t]{2}{*}{ BB FUNDO DE AÇÕES } & $(\mathbf{0 , 0 0 2 7 )}$ & $\mathbf{0 , 8 6}$ & $(0,054)$ \\
\hline & $(1,50)$ & 32,41 & $(0,25)$ \\
\hline \multirow[t]{2}{*}{ BOSTON AÇÕES } & $(0,0036)$ & $\mathbf{1 , 0 7}$ & 0,742 \\
\hline & $(2,39)$ & 48,43 & 4,16 \\
\hline \multirow[t]{2}{*}{ CCF-AÇÕES } & $(\mathbf{0 , 0 0 2 0})$ & 1,09 & 0,560 \\
\hline & $(1,13)$ & 42,13 & 2,68 \\
\hline \multirow[t]{2}{*}{ CITIAÇÕES } & $(0,0023)$ & 1,04 & 0,496 \\
\hline & $(1,48)$ & 46,35 & 2,72 \\
\hline \multirow[t]{2}{*}{ ITAUAÇÕES } & $\mathbf{0 , 0 0 0 7}$ & $\mathbf{0 , 7 3}$ & $(\mathbf{0 , 2 0 5 )}$ \\
\hline & 0.33 & 24,52 & $(0,85)$ \\
\hline \multirow[t]{2}{*}{ UNIBANCO BLUE } & $(\mathbf{0 , 0 0 1 8 )}$ & $\mathbf{0 , 9 6}$ & $\mathbf{0 , 0 2 3}$ \\
\hline & $(0,99)$ & 37,20 & 0,11 \\
\hline \multirow[t]{2}{*}{ IP PARTICIPAÇÕES } & 0,0002 & $\mathbf{0 , 3 8}$ & $\mathbf{0 , 0 8 1}$ \\
\hline & 0,08 & 9,36 & 0,24 \\
\hline \multirow[t]{2}{*}{ BRADESCO CART. LIVRE } & $(0,0042)$ & 1,08 & $\mathbf{0 , 5 7 5}$ \\
\hline & $(2,42)$ & 42,62 & 2,80 \\
\hline \multirow[t]{2}{*}{ UNIBANCO STRATEGY } & $(0,0020)$ & $\mathbf{0 , 9 7}$ & 0,406 \\
\hline & $(1,20)$ & 40,34 & 2,08 \\
\hline \multirow[t]{2}{*}{ OPPORTUNITY LÓGICA II - CL } & $\mathbf{0 , 0 0 3 3}$ & $\mathbf{0 , 8 9}$ & $\mathbf{0 , 1 7 2}$ \\
\hline & 0,89 & 16,18 & 0,39 \\
\hline
\end{tabular}

Obs.: abaixo de cada coeficiente estão as estatísticas $t$ dos mesmos. 
Tomando-se o conjunto de coeficientes estatisticamente significativos, cinco dos fundos mostram timing positivo, e nenhum deles mostra timing significativamente negativo. $\mathrm{O}$ fundo $\mathrm{CCF}$, por exemplo, passa a ter alfa negativo (embora essa estimativa não seja significativa a 5\%).

\section{Conclusóes}

Vários indicadores tentam avaliar a performance dos fundos de investimento e eventualmente resultam em valores e classificações diferentes, pois esses indicadores podem ser calculados de diferentes maneiras, além de serem adequados para contextos específicos. Quando utilizados na situação correta, eles permitem aos investidores melhorar o bem-estar proporcionado pelos seus investimentos.

O principal problema da aplicação desses indicadores vem da dificuldade de estimar corretamente seus parâmetros. Na prática, a sua estimativa costuma ser baseada em séries históricas e, conseqüentemente, supõe que o desempenho passado é um bom previsor para o futuro. Outro problema vem do fato de que as estratégias e o próprio gestor do fundo podem mudar ao longo do tempo, invalidando as análises que são baseadas em situações estáticas.

Os problemas que surgem da aplicação dessas estatísticas de performance não têm solução fácil. Aqueles que procuram bons fundos de investimento também devem estar atentos a outros indicadores, que permitam julgar se o gestor do fundo tem condição de gerar um retorno superior no futuro. Esses indicadores vão da formação acadêmica e experiência do gestor à qualificação e comprometimento da empresa gestora.

\section{Notas}

\footnotetext{
${ }^{1}$ Agradeço à Quantum_avaliação de fundos de investimento o fornecimento dos dados. Agradeço os comentários recebidos de Marco Bonomo (EPGE/FGV) e Maxim Wengert (Quantum_avaliação de fundos de investimento).

${ }^{2}$ CAPM se refere ao capital asset pricing model, desenvolvido por Sharpe (1964).

${ }^{3}$ APT se refere à arbitrage princig theory, desenvolvida por Ross (1976).

${ }^{4}$ Isso vale supondo que a taxa de retorno a cada período seja independente da taxa de retorno de outros períodos e tenha sempre a mesma distribuição de probabilidade; em estatística diz-se, nesses casos, que a taxa de retorno deve ser independente e identicamente distribuida.
} 
${ }^{5}$ Os dados foram gentilmente fornecidos pela empresa de avaliação de fundos Quantum (www.quantumfundos.com.br).

${ }^{6}$ Um retorno esperado negativo tem sentido apenas se a correlação com o resto da carteira for suficientemente negativa, de modo a permitir uma diminuição na volatilidade total, tal que compense a queda do retorno esperado da carteira.

${ }^{7}$ Essa é apenas uma aproximação, porque o diferencial de retorno (retorno esperado menos retorno sem risco) deve levar em conta a capitalização do retorno e supõe que não há correlação serial dos retornos.

${ }^{8}$ Uma descrição do que é estilo e sua aplicação ao Brasil está em Varga e Valli (1998).

${ }^{9}$ O risco sistemático é o risco do mercado, que não pode ser diversificado.

${ }^{10}$ Por exemplo, pode-se comprar contratos futuros do índice de mercado, cujo pagamento inicial é de zero, de modo que está feita a alavancagem. Por arbitragem os contratos futuros carregam uma taxa implícita igual a taxa sem risco.

\section{Referéncias Bibliográficas}

ADMATI, A. et al.

On timing and selectivity. Journal of Finance, v. 41, p. 715-730, 1986.

ADMATI, A.;

ROSS, S.

Measuring investment performance in a rational expectations equilibrium model. Journal of Business, v. 58, p. 1-26, 1985.

BODIE, Z.;

KANE, A.;

MARCUS, A.

Investment. 4. ed. New York: McGraw-Hill, 1996.
BRITO, N.

Gestão segmentada de investimentos: avaliação de desempenho e terceirização. Revista da ANBID, [s.n.], 1996.

DUARTE, A.;

HEIL, T.;

PINHEIRO, M.

Estimação da volatilidade de ativos e índices brasileiros. Resenha da BM\&F, n. 111, 1996.

DYBVIG, P.;

ROSS, S.

Differential information and performance measurement using a security market line. Journal of Finance, [s.n.], June 1985. 
HAUGEN, R. A.

Modern investment theory. 4. ed. Englewood Cliffs, NJ: Prentice-Hall, 1997.

MODIGLIANI, F.;

MODIGLIANI, L.

Risk-adjusted performance. Journal of Portfolio Management, [s.n.], Winter 1997.

SHARPE, W.

Capital asset prices: a theory of market equilibrium under conditions of risk. Journal of Finance, v. XIX, p. 425-442, Sept. 1964.
Mutual fund performance. Journal of Business, [s.n.], p. 119-138, Jan. 1966.

The sharpe ratio. Journal of Portfolio Management, [s.n.], Oct. 1994.

TREYNOR, J.;

MAZUY, M.

Can mutual funds outguess the market? Harvard Business Review, v. 44, p. 131-136, 1966.

VARGA, G.;

VALLI, M.

Análise de estilo baseada no retorno. Revista da ANBID, n. 9, dez. 1998. 